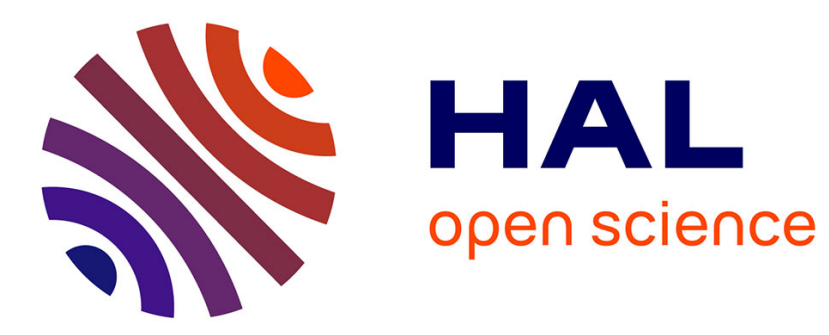

\title{
Couches minces d'oxynitrure de phosphore. Application aux structures MIS sur InP
}

\author{
Dang Tran Quan, A. Le Bloa, H. Hbib, O. Bonnaud, J. Meinnel, A. \\ Quemerais, R. Marchand
}

\section{- To cite this version:}

Dang Tran Quan, A. Le Bloa, H. Hbib, O. Bonnaud, J. Meinnel, et al.. Couches minces d'oxynitrure de phosphore. Application aux structures MIS sur InP. Revue de Physique Appliquée, 1989, 24 (5), pp.545-551. 10.1051/rphysap:01989002405054500 . jpa-00246080

\section{HAL Id: jpa-00246080 https://hal.science/jpa-00246080}

Submitted on 1 Jan 1989

HAL is a multi-disciplinary open access archive for the deposit and dissemination of scientific research documents, whether they are published or not. The documents may come from teaching and research institutions in France or abroad, or from public or private research centers.
L'archive ouverte pluridisciplinaire HAL, est destinée au dépôt et à la diffusion de documents scientifiques de niveau recherche, publiés ou non, émanant des établissements d'enseignement et de recherche français ou étrangers, des laboratoires publics ou privés. 


\title{
Couches minces d'oxynitrure de phosphore. Application aux structures MIS sur InP
}

\author{
Dang Tran Quan $\left({ }^{1}\right)$, A. Le Bloa $\left({ }^{1}\right)$, H. Hbib $\left({ }^{1}\right)$, O. Bonnaud $\left({ }^{1}\right)$, J. Meinnel ( $\left.{ }^{1}\right)$, \\ A. Quemerais $\left({ }^{2}\right)$ et R. Marchand $\left({ }^{3}\right)$ \\ (1) Département Matériaux et Composants, Groupe de Microélectronique, UA 804 CNRS, Université de \\ Rennes I, Campus de Beaulieu, 35042 Rennes Cedex, France \\ (2) Laboratoire de Spectroscopie, UA 1202 CNRS, Université de Rennes I, Campus de Beaulieu, 35042 \\ Rennes Cedex, France \\ $\left({ }^{3}\right)$ Laboratoire de Chimie des Matériaux, UA 254 CNRS, Université de Rennes I, Campus de Beaulieu, \\ 35042 Rennes Cedex, France
}

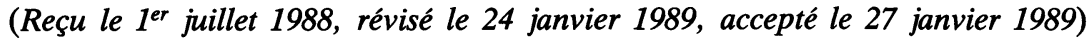

\begin{abstract}
Résumé. - Nous présentons une méthode permettant de préparer des couches minces d'oxynitrure de phosphore stables, homogènes et insensibles à l'humidité destinées à la passivation de la surface d'InP. Les couches minces ont été obtenues directement à partir du produit passivant massif (PON) déjà élaboré ; cette technique a permis de les préparer sur substrats InP aux basses températures $\left(290^{\circ} \mathrm{C}\right)$. L'analyse XPS a permis de les identifier aux composés du type $\mathrm{PO}_{x} \mathrm{~N}_{y} \mathrm{H}_{z}$ avec $1,61 \leqslant x \leqslant 1,93,0,63 \preccurlyeq y \leqslant 0,73,0,41 \preccurlyeq z \leqslant 0,75$. Les caractéristiques $C(V)$ des structures MIS présentent une hystérésis qui est de $0,20 \mathrm{~V}$ seulement pour une épaisseur d'oxynitrure de $33 \mathrm{~nm}$, ce qui traduit une bonne compatibilité entre le substrat InP et le dépôt isolant. La densité des états d'interface, dans le cas le plus favorable, atteint $5 \times 10^{11} \mathrm{~cm}^{-2} \mathrm{eV}^{-1}$.
\end{abstract}

\begin{abstract}
We present a technique for the preparation of stable, homogeneous and moisture insensitive oxynitride phosphorus films. They were fabricated directly from PON solid sample ; this technique allows for deposition on InP substrate at low temperature $\left(290^{\circ} \mathrm{C}\right)$. Using X-ray photoelectron spectroscopy these films are identified as $\mathrm{PO}_{x} \mathrm{~N}_{y} \mathrm{H}_{z}$ compounds $(1.61 \preccurlyeq x \leqslant 1.93,0.63 \preccurlyeq y \leqslant 0.73,0.41 \leqslant z \preccurlyeq 0.75)$. Electrical characteristics of MIS structures show an hysteresis at high frequency of only $0.20 \mathrm{~V}$ for $33 \mathrm{~nm}$ thick oxynitride phosphorus film, indicating structural compatibility between the insulating layer and the InP substrate. The density of the interface states, at best, is about $5 \times 10^{11} \mathrm{~cm}^{-2} \mathrm{eV}^{-1}$.
\end{abstract}

\section{Introduction.}

L'InP est un des semiconducteurs de choix pour les dispositifs microélectroniques et microoptoélectroniques [1]. Cependant les performances de ces dispositifs sont fortement limitées en raison des dégradations et instabilités de leurs caractéristiques au cours du temps. L'origine de toutes ces dégradations et instabilités n'est pas parfaitement connue mais il est vraisemblable qu'elles résultent de la facilité avec laquelle l'InP se décompose partiellement avec perte de phosphore, à des températures relativement basses. La passivation de la surface de ce composé demeure donc l'objectif prioritaire à atteindre.

Pour compenser la perte de phosphore, l'utilisation d'un isolant contenant cet élément pour passiver la surface d'InP a été essayée par plusieurs auteurs. Il a été démontré que la formation d'un oxyde riche en phosphore à la surface d'InP améliore les proprié- tés électriques des structures MIS [2-3]. Hirota et al. [4-5], Furukawa et al. [6] et plus récemment Jeong et al. [7] ont utilisé le nitrure de phosphore préparé par une méthode CVD (Chemical Vapor Deposition) pour passiver la surface d'InP. Cependant dans cette méthode, les films sont préparés sur des substrats maintenus à une température élevée $\left(>450^{\circ} \mathrm{C}\right)$ ce qui peut provoquer une détérioration de leur surface.

Nous avons tenté de passiver la surface d'InP en utilisant l'oxynitrure de phosphore (PON) dont la synthèse a été réalisée par l'un d'entre nous [8]. Ce composé, à l'état massif, est un isolant de très bonne qualité (résistivité $>10^{14} \Omega . \mathrm{cm}$ ). Par ailleurs, contrairement aux techniques CVD dans lesquelles le produit passivant est synthétisé à partir d'éléments constitutifs sur la surface du semiconducteur maintenu à haute température, notre méthode de dépôt utilise le produit passivant déjà élaboré. Elle permet 
de préparer les couches minces isolantes sur substrats InP maintenus à des températures nettement plus faibles.

Nous donnons donc dans cet article les résultats concernant la préparation de couches minces et leurs caractérisations physico-chimiques, les premières expériences de passivation et les caractéristiques des premières structures MIS sur InP.

\section{Elaboration de couches minces à partir du PON massif.}

Nous avons utilisé la technique de dépôt par transport. Le gaz vecteur est de l'azote pur circulant à faible vitesse. Le montage adopté est représenté schématiquement sur la figure 1. L'enceinte de dépôt est un tube de quartz dont une extrémité est refroidie par une circulation d'eau froide. Les différentes températures du substrat sont obtenues en faisant varier la distance «Source PON - substrat». L'azote utilisé est du type N 60 ALPHAGAZ. Pour enlever toute trace d'humidité, le courant gazeux, contrôlé par un débitmètre, traverse deux pièges à azote liquide avant de pénétrer dans l'enceinte de préparation. Une purge prolongée de l'appareil de dépôt est effectuée systématiquement avant sa montée en température. Cette montée est de l'ordre de $20^{\circ} \mathrm{C}$ par minute. Nous avons utilisé les substrats InP du type $\mathrm{n}$, d'orientation (100), non intentionnellement dopés $\left(n \sim 1-2 \times 10^{16} \mathrm{~cm}^{-3}\right)$. Ces substrats sont d'abord dégraissés au trichloréthylène, à l'acétone et à l'éthanol, et rincés à l'eau désionisée ; ils sont soumis ensuite, juste avant l'introduction dans l'enceinte de préparation, aux deux traitements chimiques à $\mathrm{HCl} 3 \mathrm{~N}$ et à $\mathrm{HF} 10 \%$ destinés à enlever la couche d'oxyde naturel résiduel présente sur leur surface.

Cette technique de préparation permet d'obtenir assez facilement des dépôts d'oxynitrure de phosphore. Cependant, les couches minces préparées sans conditions particulières sont souvent hétérogènes et sont hygroscopiques.

L'étude de la qualité et de la stabilité des dépôts en fonction des paramètres de préparation, a montré que les couches minces stables homogènes et insensibles à l'humidité sont obtenues dans les conditions suivantes :

- température de la source de PON à $920^{\circ} \mathrm{C}$;

- température du substrat supérieure à $280^{\circ} \mathrm{C}$;

— débit d'azote inférieur à 5 l.min ${ }^{-1}$.

La vitesse de dépôt est faible; elle est de l'ordre de 3-4 A. $\min ^{-1}$.

Après dépôt, la descente en température du substrat, toujours sous courant d'azote, doit être lente $\left(\sim 2^{\circ} \mathrm{C} \mathrm{min}{ }^{-1}\right)$ pour éviter la formation de craquelures dues aux contraintes thermiques. Dans notre étude, nous nous sommes limités aux températures de substrat se situant dans la gamme 250$360{ }^{\circ} \mathrm{C}$ pour ne pas provoquer une éventuelle détérioration de la surface d'InP.

La figure 2 représente la photographie du dépôt obtenue au microscope à balayage. On observe une surface homogène sans rugosité.

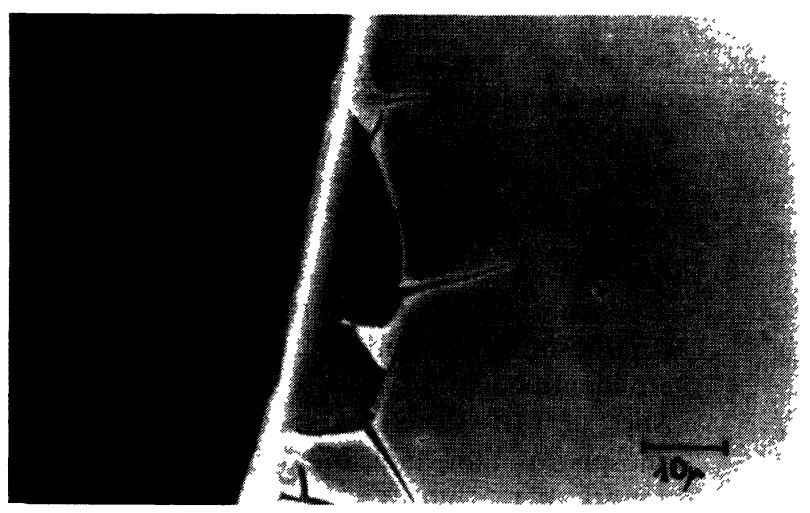

Fig. 2. - Surface de dépôt.

[Photograph of the surface of oxynitride phosphorus film.]

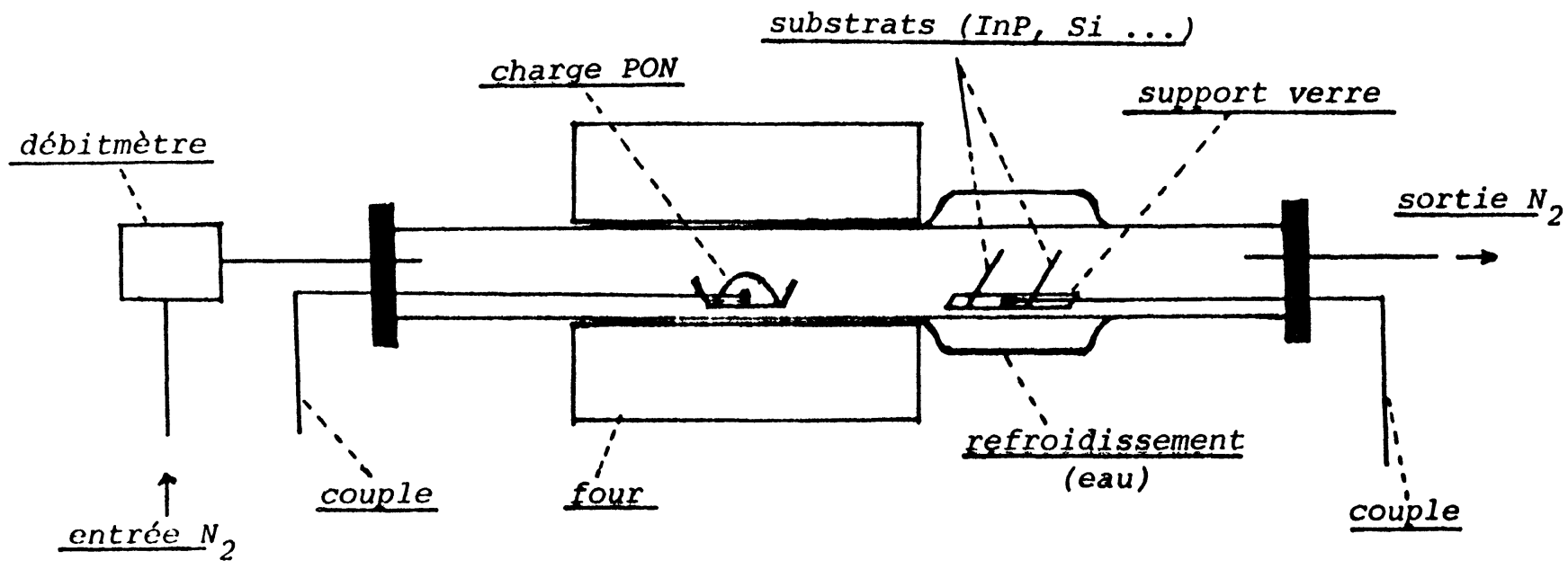

Fig. 1. - Montage de dépôt.

[Schematic diagram of the deposition apparatus.] 
Les craquelures observées à un endroit sur le bord du substrat résultent probablement d'une mauvaise adhérence du dépôt en raison de la présence, à cet endroit du substrat, d'un « défaut " de planéité ou de décapage créé lors de la coupe du substrat ou lors de son nettoyage.

\section{Caractérisations physicochimiques.}

Nous avons caractérisé les couches préparées en utilisant la spectroscopie de photoélectrons XPS. Pour les identifier, nous avons comparé les spectres obtenus à ceux du PON massif et des composés connus. Les figures 3 et 4 représentent les spectres des niveaux $\mathrm{O}_{1 \mathrm{~s}}$ et $\mathrm{N}_{1 \mathrm{~s}}$ des films minces déposés sur substrats à $340^{\circ} \mathrm{C}$ (échantillon $\mathrm{N}^{\circ} 13$ ) et à $290^{\circ} \mathrm{C}$

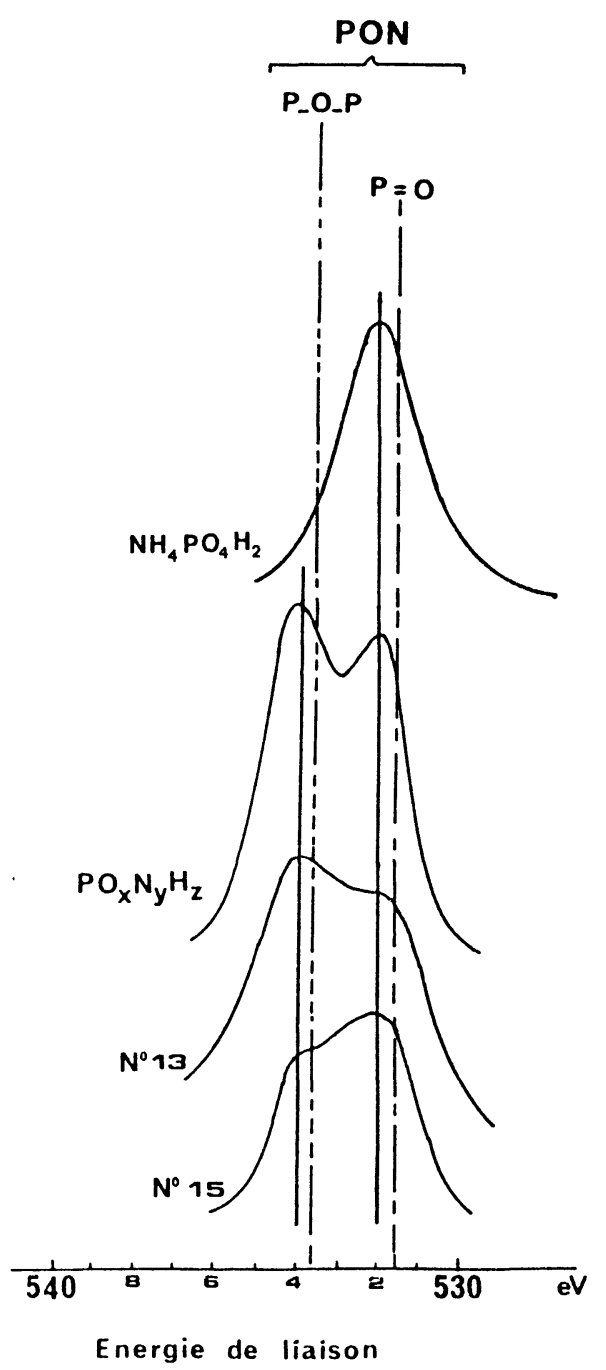

Fig. 3. - Spectres XPS des niveaux $\mathrm{O}_{1 \mathrm{~s}}$ du PON massif, des couches minces d'oxynitrure de phosphore $\left(\mathrm{N}^{\circ} 13\right.$ et $\left.\mathrm{N}^{\circ} 15\right)$, du $\mathrm{NH}_{4} \mathrm{PO}_{4} \mathrm{H}_{2}$ et du $\mathrm{PO}_{x} \mathrm{~N}_{y} \mathrm{H}_{z}(1,61 \preccurlyeq x \leqslant 1,93$, $0,63 \preccurlyeq y \preccurlyeq 0,73,0,41 \preccurlyeq z \preccurlyeq 0,75)$.

$\left[\mathrm{O}_{1 \mathrm{~s}}\right.$ spectra from $\mathrm{PON}$, oxynitride phosphorus films $\left(\mathrm{N}^{\circ} 13\right.$ and $\left.\mathrm{N}^{\circ} 15\right), \mathrm{NH}_{4} \mathrm{PO}_{4} \mathrm{H}_{2}$ and $\mathrm{PO}_{x} \mathrm{~N}_{y} \mathrm{H}_{z}, 1.61 \preccurlyeq$ $x \preccurlyeq 1.93,0.63 \preccurlyeq y \leqslant 0.73,0.41 \preccurlyeq z \preccurlyeq 0.75$.] (échantillon $\mathrm{N}^{\circ} 15$ ). (Nous n'avons pas examiné le cas des spectres $\mathrm{P}_{2 \mathrm{p}}$ car ils sont formés d'un seul pic, assez large, se situant à la même valeur d'énergie que celle du pic $P_{2 p}$ du PON massif $\left.(134,1 \mathrm{eV})\right)$. Dans chaque cas, on observe deux pics. En ce qui concerne $\mathrm{O}_{1 \mathrm{~s}}$, les pics se trouvent aux énergies $532,3 \mathrm{eV}$ et $533,9 \mathrm{eV}$. Ils sont légèrement décalés par rapport aux pics caractérisant les deux types de liaisons observés dans le $\mathrm{PON}$ massif : $\mathrm{P}=\mathrm{O}$ à $531,6 \mathrm{eV}$ et $\mathrm{P}-\mathrm{O}-\mathrm{P}$ à $533,6 \mathrm{eV}$. Les pics des couches minces semblent correspondre à ceux $(531,8 \mathrm{eV}$ et $533,9 \mathrm{eV})$ des verres de composition $\mathrm{PO}_{x} \mathrm{~N}_{y} \mathrm{H}_{z}$ que nous avons préparés à partir de $\mathrm{NH}_{3}$ et de $\mathrm{P}_{4} \mathrm{O}_{10}$ avec $1,61 \preccurlyeq x \leqslant 1,93,0,63 \preccurlyeq y \leqslant 0,73$ et $0,41 \preccurlyeq z \preccurlyeq 0,75$ [8]. Les spectres $\mathrm{N}_{1 \mathrm{~s}}$ des couches minces présentent également deux pics. Selon la

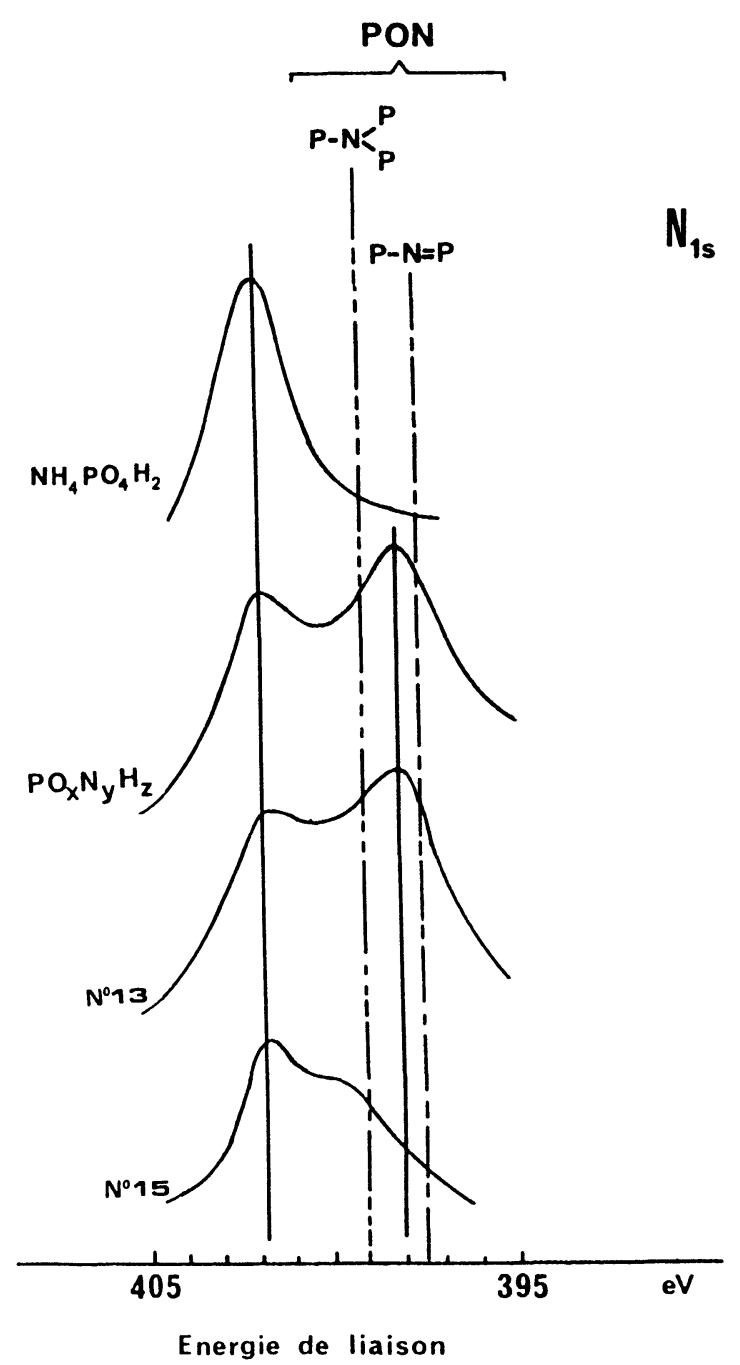

Fig. 4. - Spectre XPS des niveaux $\mathrm{N}_{1 \mathrm{~s}}$ du PON massif des couches minces d'oxynitrure de phosphore $\left(\mathrm{N}^{\circ} 13\right.$ et $\left.\mathrm{N}^{\circ} 15\right)$, du $\mathrm{NH}_{4} \mathrm{PO}_{4} \mathrm{H}_{2}$ et du $\mathrm{PO}_{x} \mathrm{~N}_{y} \mathrm{H}_{z}(1,61 \leqslant x \leqslant 1,93$, $0,63 \preccurlyeq y \preccurlyeq 0,73,0,41 \preccurlyeq z \preccurlyeq 0,75)$.

$\left[\mathrm{N}_{1 \mathrm{~s}}\right.$ spectra from PON, oxynitride phosphorus films $\left(\mathrm{N}^{\circ} 13\right.$ and $\left.\mathrm{N}^{\circ} 15\right), \mathrm{NH}_{4} \mathrm{PO}_{4} \mathrm{H}_{2}$ and $\mathrm{PO}_{x} \mathrm{~N}_{y} \mathrm{H}_{z}, 1.61 \leqslant$ $x \preccurlyeq 1.93,0.63 \preccurlyeq y \leqslant 0.73,0.41 \preccurlyeq z \preccurlyeq 0.75$.] 
température du substrat, les spectres $\mathrm{N}_{1 \mathrm{~s}}$ sont différents. Dans le cas d'une couche préparée sur substrat à $340{ }^{\circ} \mathrm{C}$, les deux pics se situent à $397,8 \mathrm{eV}$ et à $401,8 \mathrm{eV}$. L'énergie de liaison du pic «basse énergie » $(397,8 \mathrm{eV})$ n'est pas très différente de celle du pic caractérisant les liaisons de l'azote du type $\mathrm{P}-\mathrm{N}=\mathrm{P}(397,6 \mathrm{eV}) \mathrm{du}$ PON massif. Par contre, dans le cas d'une couche préparée sur un substrat maintenu à une température inférieure à $300^{\circ} \mathrm{C}$, le pic «basse énergie» semble correspondre au pic caractérisant les liaisons de l'azote du type $\mathrm{P}-\mathrm{N}<\mathrm{P}$ du PON massif. Le pic « haute énergie » $(401,8 \mathrm{eV})$ reste inchangé dans les deux cas. Ce pic correspond à une liaison de l'azote du type «ammonium ». Ici également, les pics des niveaux $\mathrm{N}_{1 \mathrm{~s}}$ correspondent à ceux des composés du type $\mathrm{PO}_{x} \mathrm{~N}_{y} \mathrm{H}_{z}$.

Le tableau I résume nos résultats et ceux de la littérature proches des nôtres. (Les valeurs des énergies des pics ont été déterminées, dans notre cas, en prenant le pic $\mathrm{C}_{1 \mathrm{~s}}$ à $285,1 \mathrm{eV}$ ).

Signalons que les spectres XPS réalisés sur une couche mince immédiatement après la préparation et après deux mois d'exposition à l'air sont identiques, ce qui montre sa stabilité.

Pour essayer de mieux identifier les couches minces obtenues, nous avons réalisé leur spectre de la bande de valence et nous les avons comparés à ceux du produit de départ et des composés connus : $\mathrm{NH}_{4} \mathrm{PO}_{4} \mathrm{H}_{2}$ et $\mathrm{PO}_{x} \mathrm{H}_{y} \mathrm{H}_{z}$. La figure 5 représente les résultats obtenus. (Dans cette figure, les spectres sont tous alignés en partant du sommet de la bande de valence). On observe la présence de plusieurs structures sur chaque spectre et une ressemblance très marquée entre eux. La similitude apparaît

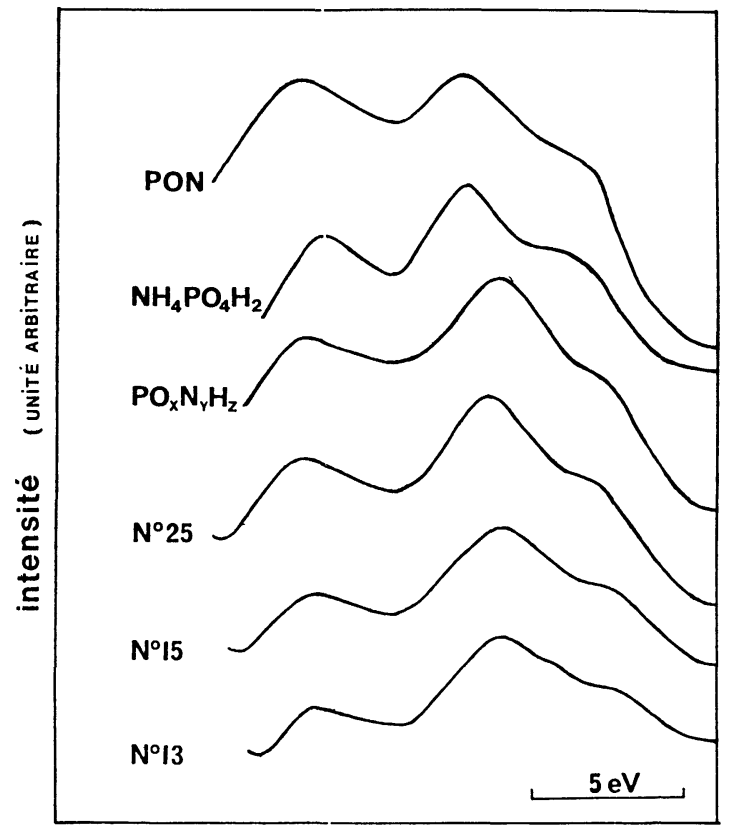

Fig. 5. - Spectres bruts des bandes de valence de PON, de $\mathrm{NH}_{4} \mathrm{PO}_{4} \mathrm{H}_{2}$, de $\mathrm{PO}_{x} \mathrm{~N}_{y} \mathrm{H}_{z}(1,61 \leqslant x \leqslant 1,93 ; 0,63 \preccurlyeq$ $y \preccurlyeq 0,73 ; 0,41 \preccurlyeq z \preccurlyeq 0,75)$ et des dépôts d'oxynitrure de phosphore ( $\mathrm{N}^{\circ} 13,15$ et 25 ).

[XPS valence band spectra (as received) for PON, $\mathrm{NH}_{4} \mathrm{PO}_{4} \mathrm{H}_{2}, \quad \mathrm{PO}_{x} \mathrm{~N}_{y} \mathrm{H}_{z}$ and oxynitride phosphorus films, Nos 13,15 and 25.]

cependant plus nette entre les spectres des couches minces ( $\mathrm{N}^{\mathrm{os}} 25,15$ et 13$)$ et les spectres de PON et des composés $\mathrm{PO}_{x} \mathrm{~N}_{y} \mathrm{H}_{z}$. Ce dernier résultat semble confirmer ceux des spectres des niveaux de cœur $\mathrm{O}_{1 \mathrm{~s}}$ et $\mathrm{N}_{1 \mathrm{~s}}$.

Tableau I. - Energie de liaison des niveaux de cour $\mathrm{O}_{1 \mathrm{~s}}$ et $\mathrm{N}_{1 \mathrm{~s}}$ des couches minces d'oxynitrure de phosphore, $d u$ PON massif et $d u \mathrm{PO}_{x} \mathrm{~N}_{y} \mathrm{H}_{z}(1,61 \preccurlyeq x \preccurlyeq 1,93,0,63 \preccurlyeq y \preccurlyeq 0,73,0,41 \preccurlyeq z \preccurlyeq 0,75)[8], d u$ $\mathrm{P}_{3} \mathrm{~N}_{5} \mathrm{H}_{1,3}$ et $d u \mathrm{NH}_{4} \mathrm{PO}_{4} \mathrm{H}_{2}$.

[Binding energies of oxynitride phosphorus films, $\mathrm{PON}, \mathrm{PO}_{x} \mathrm{~N}_{y} \mathrm{H}_{z}, \mathrm{P}_{3} \mathrm{~N}_{5} \mathrm{H}_{1.3}$ and $\mathrm{NH}_{4} \mathrm{PO}_{4} \mathrm{H}_{2}$.]

\begin{tabular}{|c|c|c|c|c|c|c|}
\hline Echantillon & $\mathrm{P}_{2 \mathrm{p}}$ & \multicolumn{2}{|c|}{$\mathrm{O}_{1 \mathrm{~s}}$} & \multicolumn{3}{c|}{$\mathrm{N}_{1 \mathrm{~s}}$} \\
\hline & & $\mathrm{P}=\mathrm{O}$ & $\mathrm{P}-\mathrm{O}-\mathrm{P}$ & $\mathrm{P}-\mathrm{N}<\mathrm{P}$ & $\mathrm{P}-\mathrm{N}=\mathrm{P}$ & $\mathrm{PO}-\mathrm{NH}_{4}$ \\
\hline $\mathrm{PON}$ massif & $134,1 \mathrm{eV}$ & $531,5 \mathrm{eV}$ & $533,6 \mathrm{eV}$ & $399,0 \mathrm{eV}$ & $397,6 \mathrm{eV}$ & \\
\hline $\mathrm{N}^{\circ} 13$ & $134,1 \mathrm{eV}$ & $532,2 \mathrm{eV}$ & $533,8 \mathrm{eV}$ & & $397,9 \mathrm{eV}$ & $401,8 \mathrm{eV}$ \\
\hline $\mathrm{N}^{\circ} 15$ & $134,1 \mathrm{eV}$ & $532,2 \mathrm{eV}$ & $533,9 \mathrm{eV}$ & $399,1 \mathrm{eV}$ & & $401,8 \mathrm{eV}$ \\
\hline $\mathrm{PO}_{x} \mathrm{~N}_{y} \mathrm{H}_{2}$ & $134,1 \mathrm{eV}$ & $531,8 \mathrm{eV}$ & $533,9 \mathrm{eV}$ & $398,6 \mathrm{eV}$ & & $401,8 \mathrm{eV}$ \\
\hline $\mathrm{P}_{3} \mathrm{~N}_{5} \mathrm{H}_{1,3}(9)$ & $133,6 \mathrm{eV}$ & & & $399,2 \mathrm{eV}$ & $397,6 \mathrm{eV}$ & \\
\hline $\mathrm{NH}_{4} \mathrm{PO}_{4} \mathrm{H}_{2}$ & & $531,8 \mathrm{eV}$ & & & & $401,8 \mathrm{eV}$ \\
\hline
\end{tabular}


A partir des intensités relatives des niveaux de cœur, nous avons déterminé les rapports atomiques $\mathrm{O} / \mathrm{P}$ et $\mathrm{N} / \mathrm{P}$ des couches préparées. Ces rapports varient d'une préparation à une autre et sont compris, dans les cas des couches stables que nous avons obtenues, entre 3 et 6 pour $\mathrm{O} / \mathrm{P}$ et entre 0,3 et 1,3 pour $\mathrm{N} / \mathrm{P}$.

La présence de deux pics sur les spectres XPS des niveaux $\mathrm{O}_{1 \mathrm{~s}}$ et $\mathrm{N}_{1 \mathrm{~s}}$ du produit de départ est due probablement au fait que le PON massif synthétisé est formé d'un mélange de deux phases : une phase amorphe assez complexe et une phase cristalline. En effet, l'étude de l'évolution des spectres des niveaux $\mathrm{O}_{1 \mathrm{~s}}$ et $\mathrm{N}_{1 \mathrm{~s}}$ du PON amorphe [10] en fonction des traitements thermiques a montré que les deux types de liaisons : $\mathrm{P}=\mathrm{O}$ et $\mathrm{P}-\mathrm{N}<_{\mathrm{P}}^{\mathrm{P}}$ qui sont prédominants dans la phase amorphe disparaissent partiellement après les traitements thermiques sous vide à $300{ }^{\circ} \mathrm{C}$ et à $550^{\circ} \mathrm{C}$. Les résultats obtenus sur les couches minces préparées sur des substrats maintenus à des températures différentes (Figs. 3, 4) semblent corroborer les conclusions des expériences sur du PON amorphe.

La présence dans les films d'oxynitrure de phosphore des liaisons correspondant à celles des composés du type $\mathrm{PO}_{x} \mathrm{~N}_{y} \mathrm{H}_{z}$ est difficilement explicable. Cependant on peut penser que ces liaisons existent en très faible nombre, dans le produit de départ du fait que la synthèse du PON fait intervenir des composés contenant à la fois l'azote et l'hydrogène. Un traitement à haute température sous azote pur et en présence d'InP augmente fortement leur nombre. Ainsi dans les couches minces d'oxynitrure de phosphore, il existe deux types de liaisons pour l'azote : liaisons du type «nitrure» et liaisons du type « ammonium ».

Il ressort ainsi que la composition atomique de ces couches est différente de celle du produit de départ et que les conditions de préparation conduisent à des dépôts de nature différente. Notons toutefois que cette différence de composition peut être acceptable dans la mesure où la couche isolante est de bonne qualité, ses propriétés de passivation sont excellentes et sa préparation est parfaitement reproductible.

\section{Elaboration et caractérisation des structures MIS.}

Les capacités MIS sont réalisées par évaporation sous vide de plots d'or de $1 \mathrm{~mm}$ de diamètre environ. Le contact ohmique est assuré par l'alliage $\mathrm{Au}-\mathrm{Ge}$ à $12 \%$ déposé également par évaporation thermique sur la face non polie de l'InP après décapage de la couche d'oxynitrure de phosphore à l'aide d'une solution d'acide fluorhydrique à $10 \%$. Les qualités électriques de la couche d'oxynitrure de phosphore sont évaluées à partir des caractéristiques $I(V)$ et $C(V)$. Les résultats que nous avons obtenus montrent que ces caractéristiques sont différentes d'une structure MIS à une autre et qu'elles dépendent étroitement des conditions de préparation de la couche d'oxynitrure de phosphore.

La figure 6 représente les variations des caractéristiques $I(V)$ à $290 \mathrm{~K}$ d'une structure MIS que nous avons réalisée avec une épaisseur d'oxynitrure de $16 \mathrm{~nm}$. On remarque que ces variations sont du même type que celles d'une Schottky améliorée. En polarisation directe, après une montée exponentielle rapide jusqu'à $0,6 \mathrm{~V}$ environ, le courant $I$ tend vers une certaine saturation. La valeur de la résistivité, au voisinage de $1 \mathrm{~V}$, est de l'ordre de $9 \times 10^{8} \Omega . \mathrm{cm}$. En polarisation inverse, le courant se sature à environ $10^{-6} \mathrm{~A}$ et le claquage se produit à $-7 \mathrm{~V}$ ce qui correspond à un champ électrique de l'ordre de $4 \times 10^{6} \mathrm{~V} . \mathrm{cm}^{-1}$.

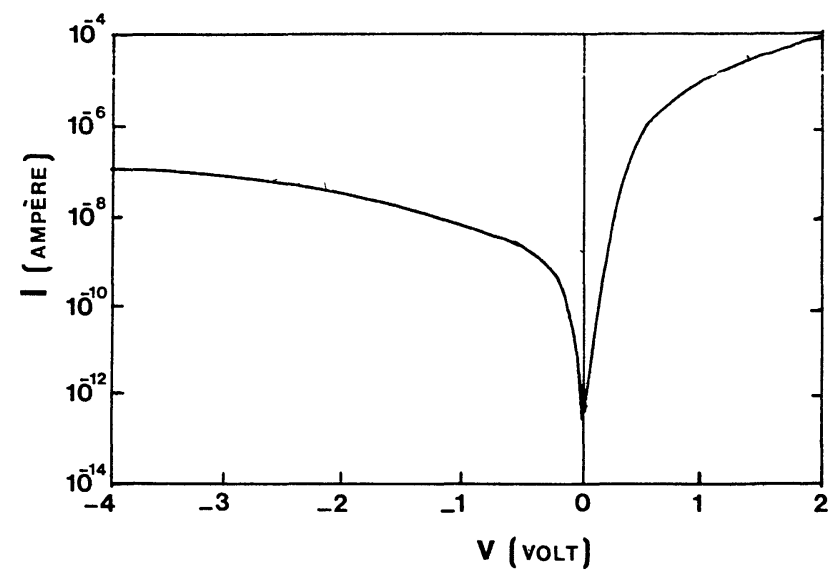

Fig. 6. - Caractéristique courant-tension d'une diode MIS oxynitrure de phosphore-InP à la température ambiante. Epaisseur de la couche d'oxynitrure de phosphore $16 \mathrm{~nm}$.

[Current-voltage characteristics of an oxynitride phosphorus-InP MIS structure. Oxynitride film thickness : $16 \mathrm{~nm}$.]

La figure 7 représente les meilleurs résultats de $C(V)$ à $1 \mathrm{MHz}$ que nous avons obtenus à la température ambiante, dans cette première phase d'études. Ils concernent les cas des structures MIS réalisées avec des couches minces d'oxynitrure de phosphore de $25 \mathrm{~nm}$ et $33 \mathrm{~nm}$ d'épaisseur sur susbtrats InP à $290{ }^{\circ} \mathrm{C}$. On observe la présence d'un cycle d'hystérésis décrit dans le sens horaire, ce qui traduit probablement une injection de charges dans la couche isolante et une présence d'états d'interface au niveau de l'interface « oxynitrure de phosphore-InP ».

La figure 8 représente les caractéristiques $C(V)$ à différentes fréquences d'une structure MIS que nous avons réalisée (échantillon $\mathrm{N}^{\circ} 25$ ). On observe une dispersion importante de la capacité dans la zone d'accumulation.

Signalons que ce phénomène se rencontre assez fréquemment dans les cas des structures MIS réali- 


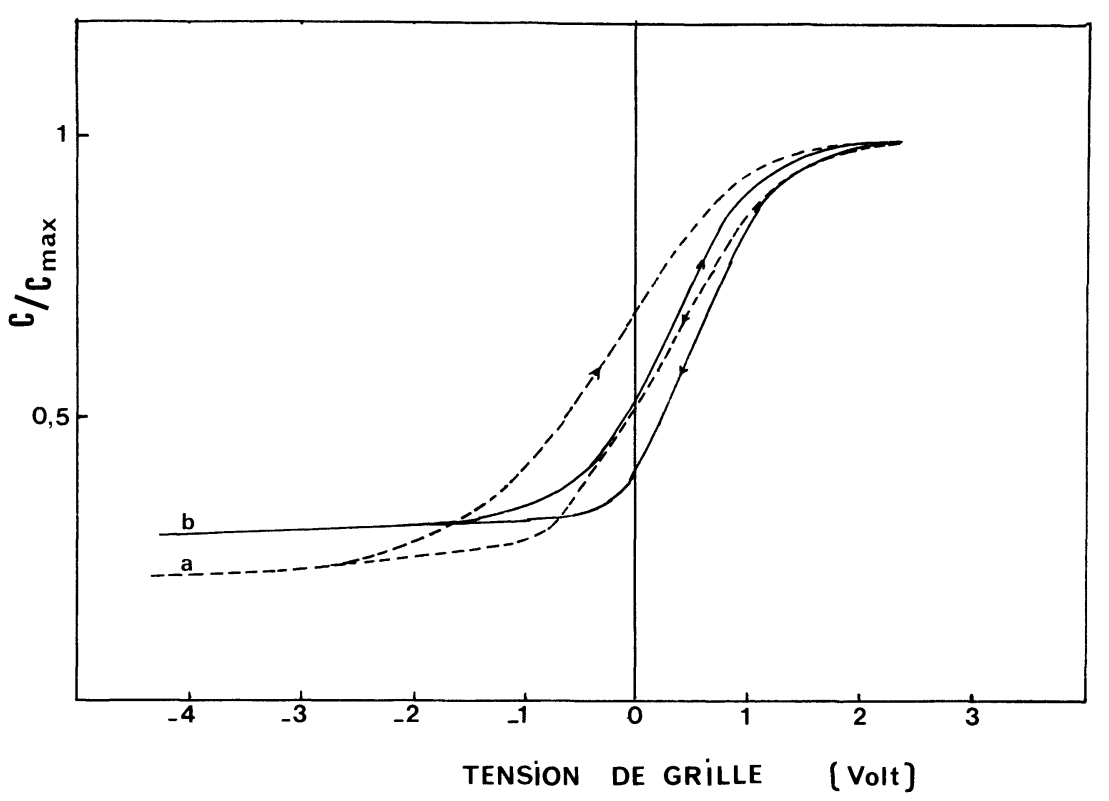

Fig. 7. - Caractéristiques capacité-tension à $1 \mathrm{MHz}$ des structures MIS oxynitrure de phosphore-InP à la température ambiante. Epaisseur de la courbe d'oxynitrure : (a) $25 \mathrm{~nm}$; (b) $33 \mathrm{~nm}$. Température du substrat : $290{ }^{\circ} \mathrm{C}$. Vitesse de balayage : $0,1 \mathrm{~V} / \mathrm{s}$.

[Capacitance-voltage caracteristics at $1 \mathrm{MHz}$ for different thicknesses of oxynitride phosphorus-InP MIS structure : (a) $25 \mathrm{~nm}$; (b) $33 \mathrm{~nm}$. Substrate temperature : $290^{\circ} \mathrm{C}$. Sweep rate : $0.1 \mathrm{~V} / \mathrm{s}$.]

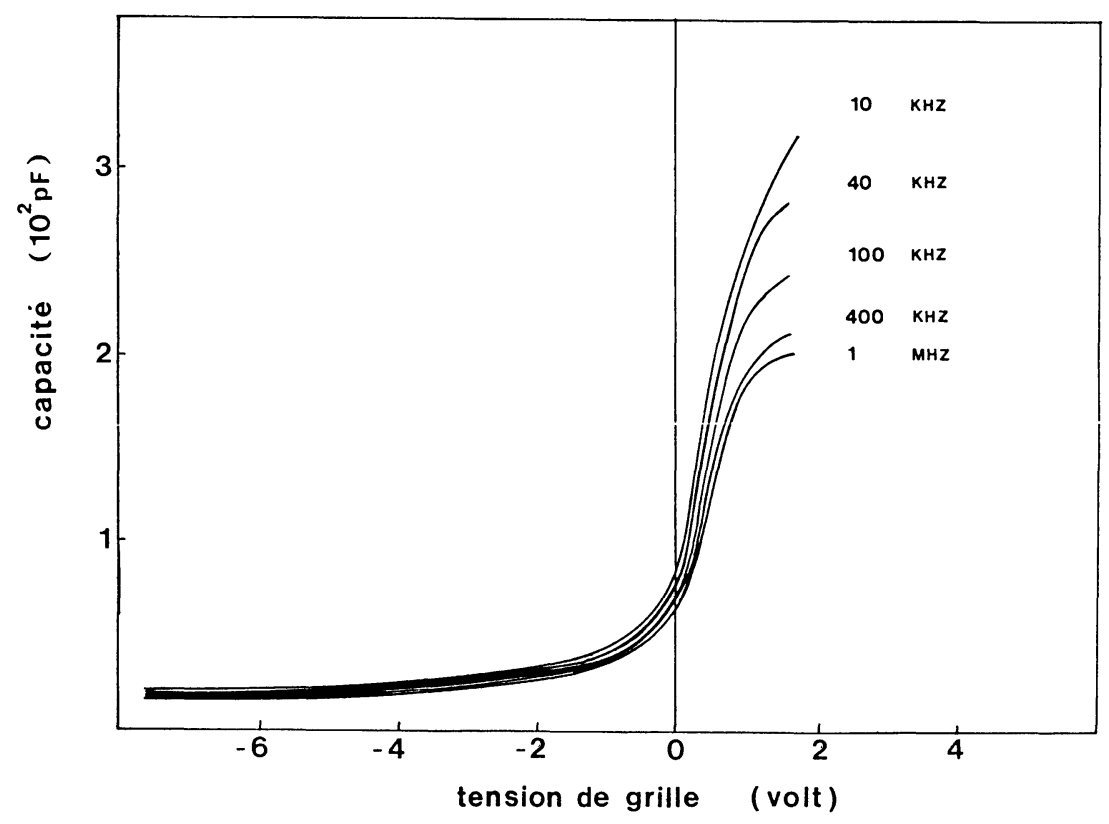

Fig. 8. - Caractéristiques capacité-tension d'une structure MIS oxynitrure de phosphore-InP à la température ambiante et à diverses fréquences. Epaisseur de la couche d'oxynitrure : $36 \mathrm{~nm}$.

[Frequency-dispersion characteristics of a oxynitride phosphorus-InP MIS structure. Oxynitride film thickness : $36 \mathrm{~nm}$.]

sées sur GaAs ou sur InP [11-18] et est souvent interprété comme lié, soit à la présence d'une forte densité d'états d'interface [12], soit à l'existence d'une conductance non négligeable dans la couche isolante $[13,17]$. Dans notre cas, la dispersion semble trop importante pour pouvoir être attribuée à la seule présence d'une forte densité d'états d'interface. Elle résulte probablement aussi du fait que la couche mince d'oxynitrure de phosphore n'est pas parfaitement isolante. En effet, la résistivité des couches préparées est de l'ordre de $10^{9} \Omega . \mathrm{cm}$, valeur très faible comparée à celles du PON massif $\left(>10^{14} \Omega . \mathrm{cm}\right)$ et du $\mathrm{SiO}_{2}$ sur Si $\left(>10^{22} \Omega . \mathrm{cm}\right)$, d'où l'existence d'un courant de fuite qui perturbe 
l'accumulation des porteurs de charges au niveau de l'interface.

La densité des états d'interface déterminée par la méthode de Terman [19], à $1 \mathrm{MHz}$, est, dans le cas le plus favorable, d'environ $5 \times 10^{11} \mathrm{~cm}^{-2} \mathrm{eV}^{-1}$.

La faible valeur de la résistivité provient vraisemblablement de la présence d'impuretés dans le PON massif. En effet dans cette première phase d'étude, ce composé n'a pas été synthétisé à partir des constituants de pureté microélectronique.

Les structures MIS préparées sont stables. Leurs caractéristiques, mesurées à plusieurs mois d'intervalle, sont restées pratiquement inchangées.

\section{Conclusion.}

Nous avons montré qu'il est possible de préparer des couches minces isolantes d'oxynitrure de phosphore à partir du PON massif par la technique de transport sous courant d'azote à la pression atmosphérique. Les couches stables, homogènes et insensibles à l'humidité, peuvent être préparées sur des substrats InP dont la température est suffisamment basse $\left(280^{\circ} \mathrm{C}-340^{\circ} \mathrm{C}\right)$ pour ne pas provoquer une détérioration de leur surface.

Les caractéristiques des premières structures MIS réalisées avec de telles couches sont assez prometteuses. La densité des états d'interface obtenue, de l'ordre de $5 \times 10^{11} \mathrm{~cm}^{-2} \mathrm{eV}^{-1}$, est comparable à celle obtenue par Jeong et al. [7] sur des structures MIS utilisant le nitrure de phosphore. Cependant, elle est plus grande par rapport à celles obtenues par Chang et al. [20] et par Robach et al. [3] dans les cas d'un oxyde du type $\operatorname{InP}_{x} \mathrm{O}_{y}$ préparé par la technique d'oxydation par plasma RF et d'un polyphosphate du type $\operatorname{In}\left(\mathrm{PO}_{3}\right)_{y}$ (avec $\left.2 \preccurlyeq y \preccurlyeq 4\right)$ préparé par la technique d'oxydation anodique.

Remarquons que dans notre étude les dépôts obtenus n'ont ni la composition ni les qualités électriques du produit de départ. Il est probable que l'utilisation du PON massif de qualité microélectronique et l'optimisation de la technique de dépôt et de la réalisation des structures MIS permettent d'améliorer la résistivité et la qualité de passivation des couches minces préparées.

\section{Remerciements.}

Nous remercions l'équipe Microélectronique de l'Ecole Supérieure d'Electricité de Rennes pour l'aide qu'elle nous a apportée pour les mesures de capacité en fonction de la fréquence.

Ce travail a été réalisé dans le cadre du contrat $\mathrm{n}^{\circ}$ 868B $068007909245 \mathrm{LAB} / \mathrm{ICM} / \mathrm{TOH}$ avec le CNET Lannion B.

\section{Bibliographie}

[1] Lile O. L., Coolens D. M., Meiners L. G. and Messich L., Electron. Lett. 14 (1978) 657.

[2] Pande K. P. and Guttierrez D., Appl. Phys. Lett. 46 (1985) 416.

[3] Robach Y., Joseph J., Bergignat E., Commere B., Hollinger G. and VikTOROVITCH P., Appl. Phys. Lett. 449 (1986) 1281.

[4] Hirota Y. and Kobayashi T., J. Appl. Phys. 23 (1984) 1157.

[5] Furukawa Y., Jpn J. Appl. Phys. 23 (1984) 1157.

[6] Hirota Y. and Mikami O., Electron. Lett. 21 (1985) 77.

[7] Jeong Y. H., Takagi S., Arai F. and Sugano T., J. Appl. Phys. 62 (1987) 2370.

[8] Marchand R., Laurent Y. and FavenneC P. N., Brevet Français CNRS/CNET N ${ }^{\circ} 8708962$;

BoukBIR L., Thèse (Rennes) 1987.

[9] Veprek S., Iqbal Z., Brunnet J. and Schark M., Philos. Mag. B 43 (1981) 527.

[10] Agliz D., Thèse (Rennes) 1987.
[11] Hirota Y. and Ковayashi T., J. Appl. Phys. 61 (1982) 257.

[12] Sawada T. and Hasegawa H., Thin Solid Films 56 (1979) 183.

[13] Sawada T. and Hasegawa H., Inst. Phys. Conf. Ser. 65 (1982) 415.

[14] Anderson W. W., Solid State Electron. 25 (1982) 1033.

[15] Ahrenkiel R. K., Sheldon P., Dunlavy D. and Roybal L., Appl. Phys. Lett. 43 (1983) 675.

[16] Sautreuil B., Bailly B., Blanchet R., Garigues M. and Viktorovitch P., Revue Phys. Appl. 18 (1983) 769.

[17] Bouchikhi B., Valmont G., Michel C. and RAVElet S., Thin Solid Films 150 (1987) 227.

[18] Klopfenstein P., Bastide G., Rouzeyre M., Gendry M. and Durand J., J. Appl. Phys. 63 (1988) 150.

[19] Terman L. M., Solid State Electron. 5 (1962) 285.

[20] Chang H. L., Meiners L. G. and Sa C. J., Appl. Phys. Lett. 48 (1986) 375. 